\title{
Reflections on the IASLC 12th World Conference on Lung Cancer
}

Rosario Perona - Bartomeu Massuti - Rafael Rosell

The 12th World Conference on Lung Cancer, organized by the International Association for the Study of Lung Cancer (IASLC), was held in Seoul, Korea on 2-6 September 2007, attaining similar success to the previous conference held in Barcelona in 2005. The proceedings were published as a supplement to the Journal of Thoracic Oncology, the official journal of the IASLC. The IASLC is a multidisciplinary society, including all aspects related to lung cancer, such as epidemiology, prevention, early diagnosis, imaging, staging, multimodality management, targeted therapies and numerous others. Since its early meetings, the IASLC has been a pioneer in including novel information on molecular biology. Prominent groups from the USA, Asia and Europe presented novel information in all areas. More than 2000 abstracts were presented, and more than 4500 investigators participated in the meeting. The participation of numerous Spanish investigators was reflected in the presentations. Due to space constraints, we will highlight only some of the Spanish presentations, with the understanding that many other important papers were also presented (see Journal of Thoracic Oncology August 2007; Vol. 2; www.jto.org). In addition, some large phase

III studies promoted by pharmaceutical firms are not discussed here, particularly as the results were not sufficiently relevant for clinical practice and associated translational research

was not performed, which makes it difficult to understand which subgroup of patients can benefit from some new therapies.

One important novelty at the conference was the presentation of the IASLC Lung Cancer Staging Project. The IASLC established this project in 1998 in order to make recommendations for the seventh edition of the TNM Classification of Malignant Tumors (to be published in 2009).

Adequate data were available on 67,725 cases of non small cell lung cancer (NSCLC) treated with all modalities of care between 1990 and 2000. The suggestions include additional cutoffs for tumour size, with tumours $>7 \mathrm{~cm}$ moving from T2 to T3. They also suggest that T2b N0 M0 cases be moved from stage IB to stage IIA, that T2a N1 M0 cases be moved from stage IIB to IIA, and that T4 N0$1 \mathrm{MO}$ cases be moved from stage IIIB to IIIA. The proposed changes would improve the alignment of TNM stage with prognosis and, in certain subsets, with treatment. Complete information on this project has been published in different articles.

Several of the sessions at the conference were related to new molecules involved in tumour development, diagnosis and treatment. John Minna presented different approaches for translating new discoveries in lung cancer pathogenesis to clinical applications. These include:

genome-wide approaches, biomarkers for early diagnosis, inherited susceptibility to lung cancer, targeted therapy, development of preclinical lung cancer systems for drug exposure

and expression profile signature development, genome-wide approaches to discovery of new therapeutic targets including synthetic lethal screens, and new discoveries on lung cancer stem cells. Ilona Linnoila gave an update on new mouse models used in lung cancer research.

These models are more complex due to the complex genetics of lung cancer and may include models comprising mutations in the K-ras oncogene and different alleles of p53 which associate with stromal metaplasias and distant metastasis characteristics of human lung adenocarcinomas.

Advances in imaging technologies applied to genetically engineered mice led to the development of new in vivo imaging techniques such as bioluminescence imaging in tumorigenesis. Finally in this session, Jerry Shay presented the characterisation of organotypic lung cultures expressing hTERT. Human bronchial epithelial cells are immortalised by expression of hTERT and cdkk4. These cells are able to differentiate into both mucous and ciliated epithelial cells when placed in 3D cultures. When these cells are transfected with KrasV12 and p53 is inactivated, they show an invasive 
phenotype, and when inoculated into the lungs of immunosuppressed mice, they develop into adenocarcinoma and squamous cell carcinoma. This indicated that human bronchial epithelial cells expressing cdk4 and hTERT fulfil the definition of cancer stem cells as they have self-renewal capacity. Results were also presented on the application of anti-telomerase strategies for cancer treatment. As activation of telomerases is a requisite for the growth of cancer stem cells, human lung spheroids were treated with the telomerase inhibitor GRN163L, and results showed a decrease in expression of stem cell markers. Therefore, it is tempting to speculate that treatment of lung tumours with GRN163L may lead to depletion of the stem cell population.

An appealing session on genetics and epigenetics in lung carcinogenesis was also presented. Steven Belinsky based his talk on the use of promoter methylation detection in different genes to be used in screening for early detection of lung cancer in smokers. A panel of 14 genes was used whose promoter methylation was detected in sputum. Six of 14 genes were associated with $>50 \%$ increase in lung cancer risk. The concomitant methylation of three or more of these six genes was associated with a 6.5 -fold increased cancer risk and a sensitivity and specificity of $64 \%$. An interesting report was also presented on the identification of genetic determinants for gene methylation based on the hypothesis that diminished DNA repair is associated with an increased methylation index.

Adi F. Gazdar used a novel method to study preneoplasias based on the introduction of tumourassociated genes into bronchial immortalised preneoplastic cultures. Introduction of Kras or p53 knockdown or both into these cells induced the development of biological features suggestive of preneoplastic respiratory epithelium, indicating that they are useful models for studying lung preneoplasia. In the prognostic factor session, Rafael Rosell presented a study on the levels of 5 transcripts of the caretaker genes ERCC1, MZF1, BRCA1, TWIST and TRX1. The samples included 126 resected NSCLCs with the aim to identify patients with a high risk of relapse. Increased BRCA1 was associated with shorter survival, indicating that BRCA1 assessment could be useful for customising adjuvant chemotherapy. (Complete information on this study has been published).

Rosario Perona presented the preliminary results of a pharmacogenomic study performed with the aim of identifying gene profiles associated with pemetrexed response in surgical samples of NSCLC. The results indicated that 168 genes were able to predict in vitro response to pemetrexed treatment with a specificity of $76 \%$ and $84 \%$ respectively for sensitive and resistant samples.

In early-stage NSCLC, Enriqueta Felip presented the Spanish Lung Cancer Group (SLCG) phase III NATCH trial, designed to address whether neoadjuvant or adjuvant paclitaxel/carboplatin improves disease-free survival compared to surgery alone in early-stage NSCLC. Six hundred and sixteen patients with clinical stage I $(>2 \mathrm{~cm})$, II, T3N1 NSCLC were randomised to surgery alone or three cycles of neoadjuvant paclitaxel $200 \mathrm{mg} / \mathrm{m} 2$ plus carboplatin AUC 6 on day 1 every 3 weeks, or surgery followed by 3 cycles of adjuvant paclitaxel plus carboplatin on the same schedule.

Major radiographic response has been observed in 59\% of patients. Median tumour size was 4.5 $\mathrm{cm}$ at baseline CT scan and $2.5 \mathrm{~cm}$ at surgery in the neoadjuvant arm. At surgery, $9 \%$ of patients had pathologic complete response; $75 \%$ N0-1 disease (with persistent T tumour), and $15 \%$ had pathologic N2 disease. Neoadjuvant chemotherapy in early NSCLC has proven feasible and safe in this large multicentre sample. Mature survival results of the NATCH trial are expected in 2009.

In locally advanced NSCLC, Pilar Garrido presented the SLCG phase II trial of concomitant chemoradiation plus induction of consolidation chemotherapy in unresectable stage IIIB NSCLC. One hundred and fifty-one patients were initially randomised to sequential treatment (arm $A$ ), concurrent chemoradiation followed by consolidation chemotherapy (arm B) or induction chemotherapy followed by concurrent chemoradiation (arm C). All patients received two cycles of docetaxel $40 \mathrm{mg} / \mathrm{m} 2$ on days 1 and 8 plus gemcitabine $1200 \mathrm{mg} / \mathrm{m} 2$ on days 1 and 8 as induction 
or consolidation therapy. Concomitant treatment was docetaxel $20 \mathrm{mg} / \mathrm{m} 2$ and carboplatin AUC 2 weekly plus 60 Gy radiation. Toxicity grade $3-4$ by CTC and RTOG criteria was: oesophagitis $19.5 \%$ (arm B) and 14.2\% (arm C); pneumonitis 8.8\% (arm B) and 10\% (arm C). Response

rates were $57 \%(\operatorname{arm~B})$ and $56.9 \%$ (arm $C$ ). A trend towards longer time to progression was observed in arm C but overall survival was similar in both arms (B: 14.3 months, C: 14.7 months; $p=0.38$ ).

Manuel Domine presented a SLCG study in stage IV NSCLC patients treated with chemotherapy, showing the potential relevance of specific single nucleotide polymorphisms (SNPs). ERCC6 (alternate name CSB) is involved in both transcription coupled and base excision DNA repair, and the ERCC6 C-6530>G SNP is involved in gene regulation. Different levels of ERCC6 mRNA expression have been observed in cells according to the ERCC6-6530 genotype. The ERCC6 C$6530>$ G SNP was examined in 309 stage IV NSCLC patients treated with docetaxel/cisplatin or gemcitabine/cisplatin. No differences in genotype were observed according to age, gender, performance status (PS), histology, chemotherapy regimen or second-line treatment. Overall time to progression was 5.4 months and median survival was 9.9 months, with no differences according to ERCC6 SNP types. However, when patients were broken down by chemotherapy regimen, time to progression $(p=0.04)$ and median survival $(p=0.46)$ were longer for CC patients treated with gemcitabine/cisplatin than for CC patients treated with docetaxel/cisplatin. ERCC6 C-6530>G SNP may confer differential sensitivity to gemcitabine or docetaxel in combination with cisplatin.

The IASLC World Conference on Lung Cancer (http://www.iaslc.org) is celebrated bi-annually, and the next conference will be held in 2009 in San Francisco, and the following in 2011 in Amsterdam. The venue for the conference alternates between Asia, North America and Europe. However, due to the rapid pace of innovation in lung cancer research, a joint lung cancer meeting will now be held annually, sponsored jointly by IASLC and the European Society of Medical Oncology (ESMO). The First European Lung Cancer Conference will be held in Geneva on 23-26 April 2008 (http://www.esmo.org).

Table 1 ERCC6 genotypes and chemotherapy outcome in stage IV NSCLC ERCC6

$\mathrm{N}$

$\mathrm{CC}$

$\mathrm{Doc} / \mathrm{Cis}$

$\mathrm{Gem} / \mathrm{Cis}$

GG

$\mathrm{Doc} / \mathrm{Cis}$

$\mathrm{Gem} / \mathrm{Cis}$

CG

$\mathrm{Doc} / \mathrm{Cis}$

$\mathrm{Gem} / \mathrm{Cis}$

56
TTP (m)

5.4

7

5.9

4

5.2

6

6

$95 \% \mathrm{Cl}$

3.1-7.7

2.5-11.4

4.3-7.5

0.4-7.6

4-6.4

4.8-7.2 $p$

0.04

0.90

0.95 